\title{
The tremendous contribution of biosciences and biotechnology for better life: research dissemination is one of the most important role to make it happen
}

\author{
Elfahmia,b,* \\ aSchool of Pharmacy, Bandung Institute of Technology, Bandung, Indonesia 40132 \\ bBiosciences and Biotechnology Research Centre, Bandung Institute of Technology, Bandung, Indonesia 40132 \\ "Editor-in-Chief of Current Research on Biosciences and Biotechnology
}

\begin{abstract}
Biosciences and biotechnology have become the interesting topics since long to be investigated in order to understand science and technology, develop many tools and instrumentation, discovery of new drugs including natural base drugs and biopharmaceutical, contribute in energy sources, interact with the environment as well as to improve the quality of human being. The researches of the field have dramatically contributed for many other field and for better life. Uncounted numbers publications in these field will be continuously rising. To contribute in dissemination of research output which have been done by researcher around the world, the Current Research on Biosciences and Biotechnology so called CRBB is published in this year. In the beginning this journal will publish 2 issue in the year and will be increased in the future. All researchers in the field of bioscience and biotechnology are invited to submit the manuscripts to CRBB. The manuscript will be peer reviewed prior to publish.
\end{abstract}

Development of sciences and technology, as well as sophisticated instruments, have stimulated the research community in the field of bioscience and biotechnology to improve the quality of research methodologies resulting in high quality research. Biosciences and biotechnology have become very interesting and one of top priorities in many research groups around the world. These researches are accelerated by recent development of massive DNA synthesis/sequencing, recombinant DNA technology, systems and synthetic biology, omics studies such as genomics, proteomics, transcriptomics, and metabolomics, CRISPR tools for genome editing, and many others. Biotechnology has significantly contributed to many areas of life such as the health, agriculture, food, environment, energy. Discovery and development of drugs, biopharmaceuticals, vaccine using recombinant DNA technology has given tremendous impact in better life and economic aspect.

Nature serves as the biological sources that can be useful for human life ranging from food to health maintaining purposes. In early life, the ancient population were looking for something in the environment or nature to be consumed and have physiological effects in humans. This has become a fundamental background for discovery of drugs from natural sources. The discovery of penicillin from Penicillium sp, morphine from opium plant, quinine and its derivatives from Chincona succirubbra are all excellent discoveries of medicine from natural sources. These finding have been continuing with many others not limited to drugs but also to food, nutraceutical, energy, etc. Understanding to all aspects of life or bioscience bring us to encourage the new innovation and technology in exploring of natural sources for the high value. Researches have contributions to understand the sciences about the nature and how to explore it into the valuable products for human being. Dissemination of research finding has opened the challenge to continuously improve the understanding in sciences. In addition to patents and other intellectual property rights, scientific journals are the most valuable way to distribute knowledge resulting from scientific research into the global area. The number of research articles tend to rise surprisingly every year, including in the field of bioscience and biotechnology. Picking an example in a period of two decades, 1993-2013, the number of pharmaceutical biotechnology papers published in 252 issues of the Journal of Pharmaceutical Sciences significantly increased. At the beginning of the period, only one biotechnology article was published. Furthermore, between 1994 and 2013, more than 1000 pharmaceutical biotechnology papers have been published. A year later, during the first 6 months, 47 additional papers had been published. This dramatic growth in pharmaceutical biotechnology papers in this journal is also shown by many prestigious scientific journals (Volkin et al., 2015). The prospect of many products from the research output on bioscience and biotechnology field for better life have become very important in the future, therefore these will be very interesting topic to be investigated by many researchers around the world.

The research community continuously need to find a way to share their research findings to the public through scientific journals. In order to contribute to the dissemination of research, it is our pleasure to publish a new scientific journal named Current Research on Biosciences and Biotechnology (CRBB). This journal is published by the Bioscience and Biotechnology Research Centre, Centre for Research and Community Services, Bandung Institute of 
Technology. In the beginning, CRBB will publish 2 issues per year, and will gradually increase the publishing frequency. In this first issue, papers present quite a broad variety of bioscience and biotechnology research findings. There are 7 papers published in this issue, consisting of 1 review paper and 7 original research papers. Three original articles have continued the investigation of secondary metabolites from different plants with potential pharmacological activities. Three article presents research findings on the bacterial lactic metabolites, and a review article addresses aspects of plant fertilization and of the phenomenon of genetic crossincompatibility in maize controlled by the Gametophyte 1 locus, leading to the understanding about the failure to accomplish a successful fertilization and a full seed set in selected plant.
The Editors have committed to provide a large effort to further establish the journal as one of the selected journals that could be chosen by scientists and researchers worldwide for publishing their scientific papers. Your contribution to publish the study design and methodology as well as the final results of your researches in CRBB are very much appreciated.

\section{References}

Volkin DB, Hershenson S, Ho RJY, Uchiyama S, Winter G, Carpenter JF. 2015. Two decades of publishing excellence in pharmaceutical biotechnology. J Pharm Sci 104(2):290-300. DOI: $10.1002 / j p s .24285$.

Gaynes R. 2017. The discovery of penicillin-new insights after more than 75 years of clinical use. Emerg Infect Dis 23(5):84953. DOI: $10.3201 /$ eid2305.161556 